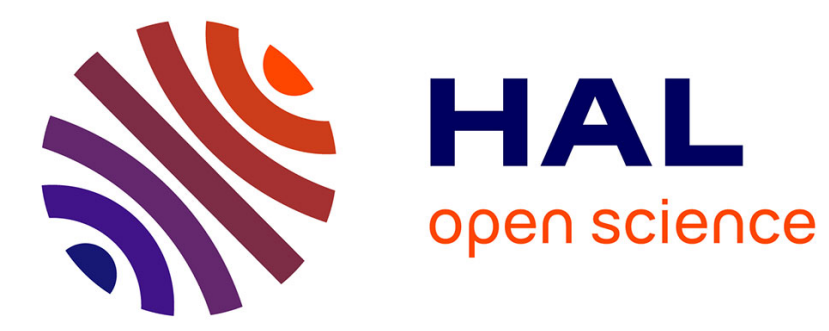

\title{
Spatial-domain interferometer for measuring plasma mirror expansion
}

M Bocoum, F Böhle, A. Vernier, Aurélie Jullien, J. Faure, R. Lopez-Martens

\section{To cite this version:}

M Bocoum, F Böhle, A. Vernier, Aurélie Jullien, J. Faure, et al.. Spatial-domain interferometer for measuring plasma mirror expansion. Optics Letters, 2015, 10.1364/OL.40.003009 . hal-01229263

\section{HAL Id: hal-01229263 \\ https://hal-ensta-paris.archives-ouvertes.fr/hal-01229263}

Submitted on 23 Nov 2015

HAL is a multi-disciplinary open access archive for the deposit and dissemination of scientific research documents, whether they are published or not. The documents may come from teaching and research institutions in France or abroad, or from public or private research centers.
L'archive ouverte pluridisciplinaire $\mathbf{H A L}$, est destinée au dépôt et à la diffusion de documents scientifiques de niveau recherche, publiés ou non, émanant des établissements d'enseignement et de recherche français ou étrangers, des laboratoires publics ou privés. 


\title{
Spatial-domain interferometer for measuring plasma mirror expansion
}

\author{
Maïmouna Bocoum, ${ }^{1, *}$ Frederik Böhle, Aline Vernier, ${ }^{1}$ Aurélie Jullien, ${ }^{1}$ Jérôme Faure, ${ }^{1}$ Rodrigo \\ Lopez-Martens, ${ }^{1}$ \\ ${ }^{1}$ Laboratoire d'Optique Appliquée, Ecole Nationale Supérieure de Techniques Avancées , Ecole Polytechnique CNRS UMR \\ 7639, 91761 Palaiseau, France \\ *Corresponding author: maimouna.bocoum@ensta-paristech.fr
}

Compiled June 8, 2015

\begin{abstract}
We present a practical spatial-domain interferometer for characterizing the electronic density gradient of laser-induced plasma mirrors with sub-30 femtosecond temporal resolution. Time-resolved spatial imaging of an intensity-shaped pulse reflecting off an expanding plasma mirror induced by a time-delayed pre-pulse, allows us to measure characteristic plasma gradients of 10 to $100 \mathrm{~nm}$ with an expansion velocitiy of $10.8 \mathrm{~nm} / \mathrm{ps}$. Spatial-Domain Interferometry (SDI) can be generalized to the ultrafast imaging of $\mathrm{nm}$ to $\mu \mathrm{m}$ size laser-induced phenomena at surfaces. (C) 2015 Optical Society of America

OCIS codes: (120.5050) Phase measurement; (110.3175) Interferometric imaging; (070.7145) Ultrafast processing; (320.7100) Ultrafast measurements.

http://dx.doi.org/10.1364/OL.99.099999
\end{abstract}

Plasma mirrors constitute an ideal testbed for studying the interaction of ultra-high intensity laser light and solid density plasmas [1]. However, the main challenge in such experiments is knowing and controlling the thin electronic density gradient that forms at the plasmavacuum interface. High-order harmonic generation experiments performed with a controlled pre-pulse [2] have allowed direct observation of the transition to the relativistic oscillating mirror regime for sharp density gradients $L / \lambda \ll 1$, where $\lambda$ is the driving laser wavelength and $L$ the density gradient length. Electron acceleration up to few- $100 \mathrm{keV}$ energies has been observed from plasma mirrors driven with density gradients increased up to a significant fraction of $\lambda[3,4]$. Short-pulse ion acceleration experiments on thin foils have shown the rapid drop of signal that occurs for leading edge intensities $\geq 10^{12} \mathrm{~W} / \mathrm{cm}^{2}$ already a few picoseconds (ps) before the pulse peak [5].

When a laser pulse of intensity $\geq 10^{12-13} \mathrm{~W} / \mathrm{cm}^{2}$ interacts with a solid surface, multiphoton ionization quickly leads to the creation of a plasma of temperature typically around $10-100 \mathrm{eV}$ [6]. The plasma inertia is dominated by its ionic constituents and its pressure by the free electrons that transmit their energy to the ions, resulting in plasma expansion velocities of the order of several $\mathrm{nm} / \mathrm{ps}$. It is therefore essential to develop simple techniques for characterizing this expansion with both sub- $\mu \mathrm{m}$ and sub-ps precision. The characteristic spatial and temporal expansion scale lengths $(<10 \mathrm{~nm},<1 \mathrm{ps}$ respectively) make femtosecond (fs) laser pulses the best candidates for performing accurate measurements using phase-sensitive detection schemes such as Frequency Domain Interferometry (FDI) [7]. Although FDI can reach $\lambda / 2000$ resolution, it is rather complex to implement on a running plasma mirror experiment. In this letter, we demonstrate a new technique we call Spatial
Domain Interferometry (SDI), easy to implement experimentally, based on time-resolved spatial phase-shift imaging interferometry using sub-30fs laser pulses. Similar to the fs pump-probe spatial imaging technique employed in $[8,9]$, our time resolution is simply limited by the duration of the pulse used to probe the plasma expansion induced by the pump pulse.

The principle of SDI applied to femtosecond plasma mirror imaging is shown on Fig 1. An intensity mask is used to generate a diffraction pattern at the focus of a time-delayed probe pulse reflecting off the surface of a plasma mirror induced on a solid target by a pump pulse (Fig 1b). The temporal resolution of this spatial interferometer system is naturally limited by the laser pulse duration $(<10 \mu \mathrm{m}$ for $30 \mathrm{fs}$ pulses at $800 \mathrm{~nm}$ wavelength). The reflection of the central diffraction spot by the peak of the plasma mirror surface induces a phase shift relative to the higher order diffraction spots around it, leading in turn to a clearly observable time-dependent spatial interference pattern in the far-field intensity profile of the reflected probe pulse. We designate as $T_{M}$ the intensity masked introduced in the collimated probe beam prior to the focusing optic of focal length $f$. As a consequence, the probe focus spot splits into several smaller spots spaced by $\Delta x=\frac{\lambda f}{a}$, where $a$ is the period of the mask. The probe field after the mask can be written as:

$$
E_{M}(x, y, \omega)=E(x, y, \omega) T_{M}(x, y) .
$$

Within the paraxial approximation, the spatial profile of the probe field in the focusing plane writes:

$$
E_{1}=F T\left[E_{M}\right]=F T\left[E(x, y, \omega) T_{M}(x, y)\right] .
$$

As shown in Fig 1(a), a plasma mirror of size $d<2 \Delta x$ induces a phase shift $\phi(x, y)$, where the central spot of 
(a) SDI basic principle

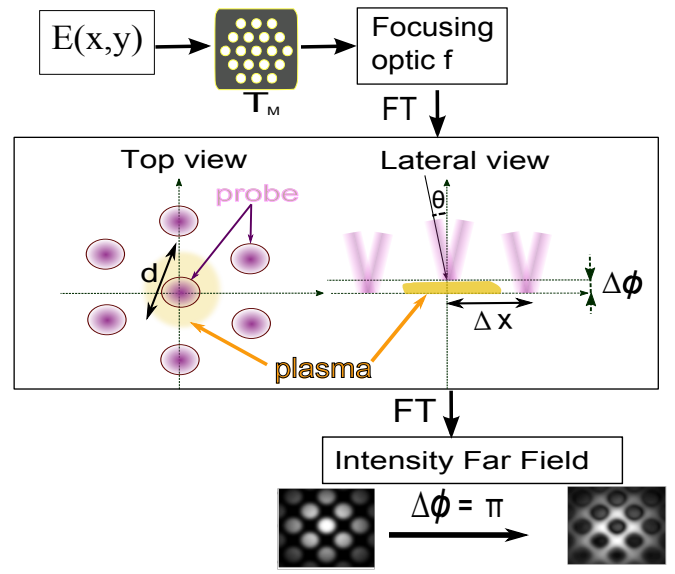

(b) Experimental set-up

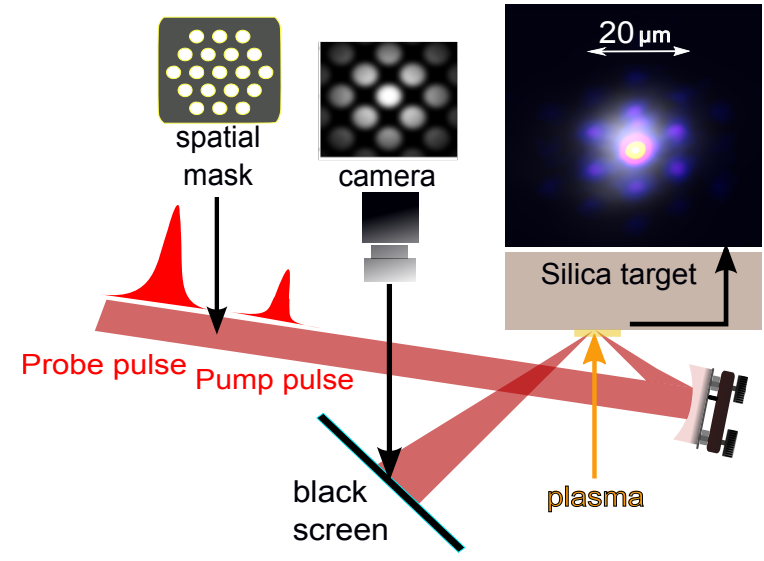

Fig. 1. (a) : SDI principle applied to ultrafast plasma mirror imaging: the probe field $E(x, y)$ is diffracted by an intensity mask $T_{M}$ onto a plasma mirror in the focal plane. The phase shift, $\Delta \phi$, of the central diffraction spot induced by the plasma expansion leads to an inversion of the far-field spatial intensity profile of the reflected probe beam for $\Delta \phi=\pi$ (b) : Experimental set-up: a $30 \mathrm{fs}$ pump pulse is focused onto a solid target using an off-axis parabola in order to induce a plasma mirror. The farfield intensity profile of the time-delayed spatially shaped probe pulse reflected by the plasma mirror is imaged onto a CCD camera and recorded as a function of pump-probe delay. The far right panel shows both pump (gray scale) and probe (color scale) beam profiles measured at focus on target.

the diffracted beam gets reflected such that we can write:

$$
E_{2}=E_{1} T=F T\left[E(x, y, \omega) T_{M}(x, y)\right] T(x, y)
$$

where, $T=|T| e^{i \phi}$, is the transfer function of the probed plasma surface. By Fourier transform of Eq 3, we obtain the reflected far field $E_{F}$ :

$$
E_{F}=F T\left[F T\left[E(x, y, \omega) T_{M}(x, y)\right]|T| e^{i \phi(x, y)}\right] .
$$

By doing a change of variables and using the relation $F T(f g)=F T(f) * F T(g)$, we can verify that the re- flected intensity will be of the form:

$I(x, y)=\left|E T_{M}+\iint E T_{M}\left(x-x^{\prime}, y-y^{\prime}\right) h\left(x^{\prime}, y^{\prime}\right) d x^{\prime} d y^{\prime}\right|^{2}$,

where

$$
h(x, y)=F T(T-1)\left(\frac{x}{\lambda f}, \frac{y}{\lambda f}\right)
$$

To estimate the effect of plasma mirror size on the reflected image, we can make the simple assumption that all diffracted spots are reflected equally by the plasma and that the $0^{\text {th }}$-order alone is phase shifted by a spatially uniform $\phi_{0}$. Mathematically, this translates into $|T|=1$ and $\phi(x, y)=\phi_{0}$ for $|x|,|y|<\frac{\lambda f}{2 a}$. This allows us to express $h$ by limiting the Fourier integration domain to $\left[-\frac{\lambda f}{2 a} \frac{\lambda f}{2 a}\right]$, such that:

$$
h(x, y)=\left(e^{i \phi_{0}}-1\right)\left(\frac{\lambda f}{a}\right)^{2} \operatorname{sinc}\left(\frac{x}{2 a}\right) \operatorname{sinc}\left(\frac{y}{2 a}\right) .
$$

This particular solution shows that the convolution function, $h$, is periodic with $\phi_{0}$ since the interference term in Eq 5 leads to an inversion of the far-field for $\phi_{0}=\pi n$ where $n$ is integer. Therefore, the monotonic expansion of the plasma mirror surface can be retrieved following each periodic inversion of the recorded interferogram. Our experiment is however different from this ideal case because the electronic density gradient at the plasma mirror surface is better represented by an exponential decay rather than by a simple step function. This, however, does not prevent the inversions from being visible in the far-field intensity pattern and each of them is associated with a $\pi$ phase shift, as shown in Fig 2, where we compare the theoretical and experimental probe beam profiles for different pump-probe delays.

Our experiment was conducted using the newly upgraded Salle Noire laser system of LOA, which delivers high-contrast, multi-mJ, $30 \mathrm{fs}$ pulses at $1 \mathrm{kHz}[10]$. All beams used in the experiment are derived from the main $30 \mathrm{fs}, 1 \mathrm{~mJ}, 15 \mathrm{~mm}$ pulses and focused using the same $f / 1.2$ off-axis parabola onto a rotating silica target at $1 \mathrm{kHz}[11]$ repetition rate. $5 \%$ of the main beam is picked off as the prepulse, reduced in size by a factor of 3 with a reflecting telescope and sent through a variable delay stage prior to focusing. The remainder of the main pulse is used as the probe pulse that is sent through an $a=4 \mathrm{~mm}$ periodic hexagonal mask with holes of $2.5 \mathrm{~mm}$, leading to diffracted spots at the focus on target as shown in Fig 1b, which compares typically measured pump (gray scale) and probe (color scale) beam profiles. The central diffracted probe spot intensity is $\sim 10^{16} \mathrm{~W} / \mathrm{cm}^{2}$ which is about 2 orders of magnitude less than without the mask such that the laser contrast plays a negligeable role in the plasma dynamic. This allows to keep the set-up still for high field experiments by simply removing the mask. The peak intensity of the pump pulse is $I_{p, 1}=3.5 \times 10^{14} \mathrm{~W} / \mathrm{cm}^{2}$ where it 
overlaps with the central diffraction spot and on average $I_{p, 2}=8.7 \times 10^{13} \mathrm{~W} / \mathrm{cm}^{2}$ where it overlaps with the first order diffraction spots.

We image the far-field spatial profile of the reflected probe beam with a CCD camera, integrated over 100 shots for every pump-probe delay to compensate for a low individual signal shot, on an anodized black screen placed in the specular direction. The results for four different pump-probe delays are displayed in the right column of Fig 2. We take the intensity profile recorded at zero pump-probe delay as our reference. The first inversion of the intensity profile appears at $12 \mathrm{ps}$ and the second inversion occurs at $\simeq 20$ ps. The profile inversion patterns are well reproduced by the simulations shown on the left column of Fig 2 where we used the actual experimental pump pulse profile to simulate the phase shift due to the plasma expansion. We describe hereafter the model used to simulate the plasma expansion dynamics and extract the density gradient and its expansion velocity.

The electron density gradient is commonly derived from the self-similar solution of the isothermal equation of state for the electron [6] and is expressed by a decreasing exponential:

$$
n_{e}(x)=n_{c} \exp \left(-\frac{x-x_{c}(t)}{c_{s} t}\right),
$$

where $c_{s}=\left(Z k_{b} T_{e} / m_{i}\right)^{1 / 2}$ is the ion sound velocity and $n_{c}=\left(\omega_{0}^{2} m_{e} \epsilon_{0}\right) / e^{2}$ the critical density. In these expressions, $Z$ is the ion charge state, $e$ is the electron charge, $k_{b}$ the Helmholtz constant, $T_{e}$ the electron temperature, $m_{e}$ and $m_{i}$ respectively the electron and ion mass and $\omega_{0}$ is the carrier frequency of the probe pulse. We define $L(t)=c_{s} t$ as the gradient length, proportional to the coordinate of critical density $x_{c}(t)$. For a fully ionized silica target, the maximum density is about $300 n_{c}$, such that $x_{c}(t)=\ln (300) \times L(t) \approx 5.7 \times L(t)$.

Assuming the electron temperature, $T_{e}$, depends linearly on the energy deposited on target when the plasma is created, i.e. on the pump pulse intensity $I_{p}$, we can write, according to its definition, that $c_{s} \propto \sqrt{I_{p}}$. The pump intensity is considered to be constant for each isolated diffracted probe spot in order to define a single corresponding plasma expansion velocity. The pump intensity is $I_{p, 1}$ at the center and $I_{p, 2}$ at the location of the first order diffracted spots. This means that the center of the plasma expands nearly twice as fast as its edges. Taking into account the angle of incidence of $\theta=49.3 \mathrm{deg}$ from the target normal, we express the phase difference $\Delta \phi$ as a function of the relative difference in position of the critical surface $\Delta x_{c}$ :

$$
\Delta \phi(t)=\frac{4 \pi}{\lambda} \Delta x_{c}(t) \cos (\theta)=\frac{4 \pi}{\lambda} 0.7 \Delta x_{c}(t),
$$

where

$$
\Delta x_{c}(t)=c_{s}\left(1-\sqrt{\frac{I_{p, 2}}{I_{p, 1}}}\right) t .
$$

simulation
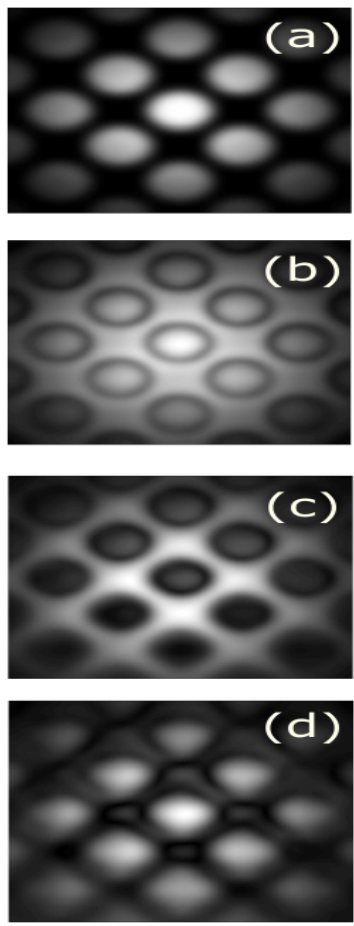

Fig. 2. Simulated (left column) and experimental (right column) far-field probe beam intensity profile inversions at respectively $0 \mathrm{ps}(\mathrm{a}) 6 \mathrm{ps}(\mathrm{b}), 12 \mathrm{ps}(\mathrm{c})$ and $20 \mathrm{ps}(\mathrm{d})$ pump-probe delay. The visible shadow is an experimental artefact. The simulations are performed using the measured pump beam profile and assuming the plasma expansion velocity to be proportional to the square root of the pump pulse intensity intensity. The corresponding phase shifts in $\operatorname{Rad}$ are respectfully $0(\mathrm{a}), \pi / 2(\mathrm{~b}), \pi(\mathrm{c})$ and $2 \pi(d)$

A linear fit of each inversion data point is performed in Fig 3, resulting in a measured expansion velocity $c_{s}=10.8 \mathrm{~nm} / \mathrm{ps} \pm 1.1 \mathrm{~nm} / \mathrm{ps}$ for a pump intensity of $I_{p, 1}=3.5 \times 10^{14} \mathrm{~W} / \mathrm{cm}^{2}$. This order of magnitude matches other measurements obtained by FDI [2], assuming the plasma expansion velocity scales as $c_{s} \propto \sqrt{I_{p}}$ and does not vary with time. The price to pay for such a simple measurement technique is an increasing error bar on the inversion as pump-probe delay increases. In order to probe longer gradients, one idea would be to decrease the period $a$ of the diffraction mask in order to increase the distance between the different diffracted spots on target and therefore be less sensitive to the first order linear phase induced by the slope of the critical plasma surface distribution. In our case, we focused on the early stages of plasma expansion $(L<\lambda)$ since this is the regime where ultrafast nonlinear dynamics of interest can occur.

In conclusion, we demonstrate that simple imaging of a spatially shaped probe pulse reflecting off an expanding 


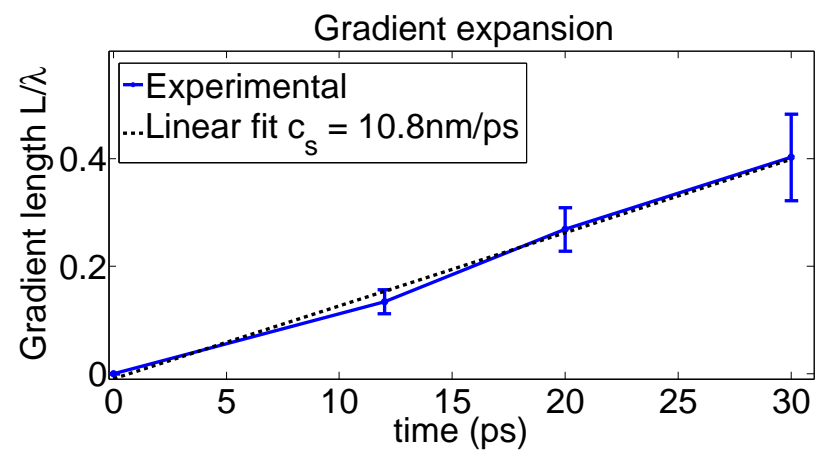

Fig. 3. Measured relative plasma expansion velocity for a pump pulse intensity of $I_{p, 1}=3.5 \times 10^{14} \mathrm{~W} / \mathrm{cm}^{2}$ as a function of pump-probe delay. Each experimental point corresponds to an inversion in the far-field probe beam intensity profile induced by the expanding critical surface of the plasma formed at the surface of the solid target. Inversions are defined by $\Delta \phi=n \pi$ with here $n=0,1,2,3$.

plasma mirror induced by a time-delayed pump pulse yields the electronic density gradient of the plasma mirror with sub- $\mu \mathrm{m}$ and sub-ps accuracy. The only assumption made here is that the plasma expansion velocity is proportional to the pump intensity [6]. As a consequence, experimental plasma expansion velocities of $\approx 10.8 \mathrm{~nm} / \mathrm{ps}$ were measured for a peak pump pulse intensity of $3.5 \times 10^{14} \mathrm{~W} / \mathrm{cm}^{2}$. This is possible because of the clearly visible far-field probe beam pattern inversions for every $\pi$ phase shift for short pump-probe delays. This is no longer the case at longer delays ( $\gg 10 \mathrm{ps},[12])$, where we reach the spatial coherence length $(\sim 6 \mu \mathrm{m})$ of our interferometer. However, our approach is accurate for very steep gradients $<\lambda$, for which efficient highorder harmonic generation and electron acceleration is typically observed from plasma mirrors. We emphasize on the simplicity of the SDI technique, which only requires inserting a spatial mask into the main beam used to drive nonlinear plasma mirror dynamics. In principle, SDI can be used for ultrafast imaging of any $\mathrm{nm}$ to $\mu \mathrm{m}$ size moving phase objects in a configuration suited to multiple-beam reflection interferometry [13]. We are currently working on a phase retrieval algorithm that would allow single-shot retrieval of the full complex probe pulse field transmitted by the plasma mirror optical system, whatever its state. This would allow 2D mapping of the both the reflectivity and phase of the expanding plasma mirror without the need for any assumption on its expansion dynamics.

This work was funded by the Agence Nationale pour la Recherche under contract ANR-11-EQPX-005ATTOLAB and the European Research Council under Contract No. 306708, ERC Starting Grant FEMTOELEC.

\section{References}

1. C. Thaury, F. Quéré, J.P. Geindre, A. Levy, T. Ceccotti, P. Monot, M. Bougeard, F. Réau, P. D'Oliveira, P. Audebert, R. Marjoribanks and Ph. Martin, Plasma mirrors for ultrahigh-intensity optics Nature Physics, 3, 424-429, 2007.

2. S Kahaly, S Monchocé, H Vincenti, T Dzelzainis, B Dromey, M Zepf, Ph Martin, and F Quéré. Direct observation of density-gradient effects in harmonic generation from plasma mirrors. Physical review letters, 110(17):175001, 2013.

3. S Bastiani, A Rousse, JP Geindre, P Audebert, C Quoix, G Hamoniaux, A Antonetti, and J-C Gauthier. Experimental study of the interaction of subpicosecond laser pulses with solid targets of varying initial scale lengths. Physical Review E, 56(6):7179, 1997.

4. Aghapi G Mordovanakis, James Easter, Natalia Naumova, Konstantin Popov, Paul-Edouard MassonLaborde, Bixue Hou, Igor Sokolov, Gérard Mourou, Igor V Glazyrin, Wojciech Rozmus, et al. Quasimonoenergetic electron beams with relativistic energies and ultrashort duration from laser-solid interactions at $0.5 \mathrm{khz}$. Physical review letters, 103(23):235001, 2009.

5. Alessandro Flacco, François Sylla, Mina Veltcheva, M Carrié, R Nuter, E Lefebvre, D Batani, and Victor Malka. Dependence on pulse duration and foil thickness in high-contrast-laser proton acceleration. Physical Review E, 81(3):036405, 2010.

6. William L. Kruer. The physics of laser plasma interactions((book)). Reading, MA, Addison-Wesley Publishing Co.(Frontiers in Physics., 73, 1988.

7. JP Geindre, A Mysyrowicz, A Dos Santos, P Audebert, A Rousse, G Hamoniaux, A Antonetti, F Falliès, and JC Gauthier. Frequency-domain interferometer for measuring the phase and amplitude of a femtosecond pulse probing a laser-produced plasma. Optics letters, 19(23):1997-1999, 1994.

8. MC Downer, Richard L Fork, and Charles V Shank. Femtosecond imaging of melting and evaporation at a photoexcited silicon surface. JOSA B, 2(4):595-599, 1985.

9. Vasily V Temnov, Klaus Sokolowski-Tinten, Ping Zhou, and Dietrich von der Linde. Ultrafast imaging interferometry at femtosecond-laser-excited surfaces. JOSA B, 23(9):1954-1964, 2006.

10. Aurélie Jullien, Aurélien Ricci, Frederik Böhle, JeanPhilippe Rousseau, Stéphanie Grabielle, Nicolas Forget, Hermance Jacqmin, Brigitte Mercier, and Rodrigo Lopez-Martens. Carrier-envelope phase stable, highcontrast, double-cpa laser system.

11. A Borot, D Douillet, G Iaquaniello, $\mathrm{T}$ Lefrou, $\mathrm{P}$ Audebert, J.P. Geindre, and R. Lopez-Martens, Review of Scientific Instruments 85, 013103 (2014) High repetition rate plasma mirror device for attosecond science, Review of Scientific Instruments, 85, 013103, 2014.

12. Thomas Kiefer, Theodor Schlegel, and Malte C Kaluza. Plasma expansion into vacuum assuming a steplike electron energy distribution. Physical Review E, 87(4):043110, 2013.

13. J Holden. Multiple-beam interferometry: intensity distribution in the reflected system. Proceedings of the Physical Society. Section B, 62(7):405, 1949. 


\section{References}

1. C. Thaury, F. Quéré, J.P. Geindre, A. Levy, T. Ceccotti, P. Monot, M. Bougeard, F. Réau, P. D'Oliveira, P. Audebert, R. Marjoribanks and Ph. Martin, Plasma mirrors for ultrahigh-intensity optics Nature Physics, 3, 424-429, 2007.

2. S Kahaly, S Monchocé, H Vincenti, T Dzelzainis, B Dromey, M Zepf, Ph Martin, and F Quéré. Direct observation of density-gradient effects in harmonic generation from plasma mirrors. Physical review letters, 110(17):175001, 2013.

3. S Bastiani, A Rousse, JP Geindre, P Audebert, C Quoix, G Hamoniaux, A Antonetti, and J-C Gauthier. Experimental study of the interaction of subpicosecond laser pulses with solid targets of varying initial scale lengths. Physical Review E, 56(6):7179, 1997.

4. Aghapi G Mordovanakis, James Easter, Natalia Naumova, Konstantin Popov, Paul-Edouard MassonLaborde, Bixue Hou, Igor Sokolov, Gérard Mourou, Igor V Glazyrin, Wojciech Rozmus, et al. Quasimonoenergetic electron beams with relativistic energies and ultrashort duration from laser-solid interactions at $0.5 \mathrm{khz}$. Physical review letters, 103(23):235001, 2009.

5. Alessandro Flacco, François Sylla, Mina Veltcheva, M Carrié, R Nuter, E Lefebvre, D Batani, and Victor Malka. Dependence on pulse duration and foil thickness in high-contrast-laser proton acceleration. Physical Review E, 81(3):036405, 2010.

6. William L. Kruer. The physics of laser plasma interactions((book)). Reading, MA, Addison-Wesley Publishing Co.(Frontiers in Physics., 73, 1988.

7. JP Geindre, A Mysyrowicz, A Dos Santos, P Audebert, A Rousse, G Hamoniaux, A Antonetti, F Falliès, and JC Gauthier. Frequency-domain interferometer for measuring the phase and amplitude of a femtosecond pulse probing a laser-produced plasma. Optics letters, 19(23):1997-1999, 1994.

8. MC Downer, Richard L Fork, and Charles V Shank. Femtosecond imaging of melting and evaporation at a photoexcited silicon surface. JOSA B, 2(4):595-599, 1985.

9. Vasily V Temnov, Klaus Sokolowski-Tinten, Ping Zhou, and Dietrich von der Linde. Ultrafast imaging interferometry at femtosecond-laser-excited surfaces. JOSA B, 23(9):1954-1964, 2006.

10. Aurélie Jullien, Aurélien Ricci, Frederik Böhle, JeanPhilippe Rousseau, Stéphanie Grabielle, Nicolas Forget, Hermance Jacqmin, Brigitte Mercier, and Rodrigo Lopez-Martens. Carrier-envelope phase stable, highcontrast, double-cpa laser system.

11. A Borot, D Douillet, G Iaquaniello, T Lefrou, P Audebert, J.P. Geindre, and R. Lopez-Martens, Review of Scientific Instruments 85, 013103 (2014) High repetition rate plasma mirror device for attosecond science, Review of Scientific Instruments, 85, 013103, 2014.

12. Thomas Kiefer, Theodor Schlegel, and Malte C Kaluza. Plasma expansion into vacuum assuming a steplike electron energy distribution. Physical Review E, 87(4):043110, 2013.

13. J Holden. Multiple-beam interferometry: intensity distribution in the reflected system. Proceedings of the Physical Society. Section B, 62(7):405, 1949. 